\title{
La Seconde Sophistique et l'Antiquité tardive
}

\author{
Laurent Pernot \\ Université de Strasbourg II \\ France
}

Resumo. A Segunda Sofística e a Antigüidade tardia. Como os estudiosos ainda não tentaram estabelecer ligações entre a Segunda Sofística e a Antigüidade tardia, é preciso abrir um caminho, questionando-se em que medida o conceito de Antigüidade tardia pode ajudar a compreender de forma nova a Segunda Sofística. As duas principais vertentes da pesquisa sobre a Segunda Sofística consistiram, por um lado, em pôr em destaque a cultura dos sofistas e suas relações com o passado grego da época arcaica e clássica e, por outro, sublinhar a importância do papel político e social que os sofistas tiveram em sua época. A terceira vertente, que é proposta aqui, permite fazer surgir, na cultura e nas concepções dos sofistas dos séculos I-III d.C., as marcas que anunciam a Antigüidade tardia e que eram, na época, portadoras do futuro. A Segunda Sofística não se limitava à admiração pelo passado e pelas funções oficiais exercidas pelos sofistas. Ela comportava também formas de devoção íntimas e exacerbadas, experiências de misticismo, um modelo de "holy man" dotado de poderes sobrenaturais, toda uma cultura filosófico-religiosa, relações com o platonismo, com o judaísmo e o cristianismo, e ainda uma prática literária rica e diversificada, que se estende, para além do discurso retórico, à poesia, à auto-biografia, à epistolografia, à narração dos sonhos, ao romance.

Palavras-Chave. Élio Aristides; Antigüidade tardia; literatura; filosofia; religião; retórica; Segunda Sofística; Tito Flávio Glauco de Maratona.

Le présent article a pour but d'examiner les relations qu'on est en droit d'instaurer entre la Seconde Sophistique et l'Antiquité tardive. La mise en rapport de ces deux notions ne va pas de soi, car, au premier abord, elles évoquent des réalités historiques et culturelles différentes. La notion de Seconde Sophistique renvoie au monde grec de la fin du ${ }^{\text {er }}$ jusqu'au III ${ }^{e}$ siècle après J.-C. (spécialement du II siècle) et à l'apogée de l'Empire païen. La notion d'Antiquité tardive, quant à elle, renvoie à la période des $\mathrm{III}^{\mathrm{e}}-\mathrm{VII}{ }^{\mathrm{e}}$ siècles, à un âge de de mutations dans tous les domaines, social, politique, artistique, religieux... D'un côté, la stabilité de l'époque antonine sous le règne des empereurs philhellènes; de l'autre, les bouleversements d'une période longue et complexe. D'un côté l' "été indien" de l'Empire; de l'autre, les "tempêtes hivernales", selon l'image de l'historien anglais Toynbee'. On peut avoir l'impression, en effet, d'un décalage chronologique et culturel très important. 
Pareil décalage explique que les chercheurs n'aient guère songé à jeter des ponts entre les deux réalités qui nous occupent. Les ouvrages consacrés à la Seconde Sophistique s'interrompent généralement à la fin de la dynastie des Sévères, les époques suivantes n'étant pas considérées comme entrant dans le champ de l'enquête. Inversement, les panoramas consacrés à l'Antiquité tardive ne prennent pas en compte la Seconde Sophistique. Tel est le cas, par exemple, du livre célèbre de H.-I. Marrou, Décadence romaine ou Antiquité tardive? (III ${ }^{e}-V I^{e}$ siècle)? ${ }^{2}$, du fascicule méthodologique d'Av. Cameron intitulé L'Antiquité tardives, ou encore du récent volume collectif Late Antiquity. A Guide to the Postclassical World, publié à Harvard par G. Bowersock, P. Brown et O. Grabar ${ }^{4}$. Dans aucun de ces ouvrages n'est étudiée ni même citée la Seconde Sophistique. Si, dans d'autres études, certains historiens ont pris en compte le $\mathrm{II}^{\mathrm{e}}$ siècle alors qu'ils traitaient de l'Antiquité tardive, leur propos n'était pas, en élargissant ainsi la définition de la période, d'en tirer des conséquences pour l'évaluation des phénomènes littéraires.

Le but de la présente recherche est donc d'ouvrir une piste, en posant la question de savoir dans quelle mesure le concept d'Antiquité tardive peut servir à interpréter de manière nouvelle la Seconde Sophistique. Le bénéfice escompté de cette démarche est une meilleure compréhension de la Seconde Sophistique, qui consistera à mettre ce phénomène en rapport avec la période qui l'a suivi, ce qu'on ne fait pas d'habitude, et à reconnaître dans la Seconde Sophistique des caractéristiques qui annoncent déjà les évolutions ultérieures. Corrélativement, cette démonstration peut apporter aussi une contribution à l'enrichissement des points de vue sur l'Antiquité tardive ellemême, en mettant l'accent sur des éléments de continuité entre Haut-Empire et Bas-Empire, et donc sur la continuité de l'hellénisme, de façon à refuser les séparations artificielles et à insister davantage sur les phénomènes de longue durée dans l'histoire de la culture.

Par "Seconde Sophistique", entendons ce phénomène littéraire et social qui, sous le Haut-Empire, dans les provinces hellénophones, a consisté dans la multiplication des "sophistes", au sens de professeurs de rhétorique, conférenciers et hommes publics. Sans entrer dans le détail des problèmes de définition, nous nous référons à l'image de la Seconde Sophistique qui se

${ }^{1}$ A.J. Toynbee, A Study of History, V (1939), rééd. New York - Oxford 1962, p. 343 (C, I, (c), 4): "In the history of the Hellenic Society the Age of the Antonines - though this was but a brief 'Indian Summer' which might delay, but could not avert, the onset of the winter storms -..."; cf. ibid., IV, p. 58-61 (C, II, (b), 1).

${ }^{2}$ Paris, 1977.

${ }^{3}$ Tr. fr. par M. Pouteau, éd. Mentha, 1992.

${ }^{4}$ Cambridge Mass.- Londres, 1999. 
reflète dans les Vies des sophistes de Philostrate, en tenant compte des autres sources que constituent les œuvres conservées des sophistes de l'époque et les inscriptions.

Quant à la deuxième notion qui entre en jeu, l'Antiquité tardive, nous ne nous aventurerons certes pas à en donner une définition générale, et c'est chemin faisant que nous essaierons de faire apparaître aussi clairement que possible, sur chaque point, ce qui est envisagé sous ce terme.

Nous rappellerons brièvement les deux principales directions actuelles de la recherche sur la Seconde Sophistique, avant de développer plus longuement la troisième direction, celle qui est ouverte par le rapprochement avec l'Antiquité tardive.

\section{La Seconde Sophistique et la mimêsis}

Une première direction de la recherche sur la Seconde Sophistique a consisté à mettre en relief la culture des sophistes et leurs références au passé grec de l'époque archaïque et classique. Cette approche est illustrée notamment par le livre de J. Bompaire, Lucien écrivain. Imitation et création, et par l'article de E. Bowie sur "Greeks and their Past in the Second Sophistic", qui date de $1970^{6}$. Ces savants, et beaucoup d'autres à leur suite, ont montré combien la Seconde Sophistique était conservatrice et tournée vers le passé. Son traditionalisme s'exprimait non seulement dans les exercices d'école (progumnasmata, déclamations), mais dans toutes les productions rhétoriques et littéraires, qui déployaient un arsenal de références empruntées en particulier à la culture athénienne des $\mathrm{V}^{\mathrm{e}}$ et $\mathrm{IV}^{\mathrm{e}}$ siècle avant J.-C. C'est la Seconde Sophistique de l'atticisme, de la paideia, de la mimêsis.

\section{Les Sophistes en leur Temps}

Parallèlement à cette première approche, et en partie en réaction contre elle, une deuxième direction de la recherche a consisté à souligner l'importance du rôle politique et social joué par les sophistes en leur temps. Cette voie a été ouverte en particulier par L. Robert, dans plusieurs passages de son œuvre 7 , par G. Bowersock dans Greek Sophists in the Roman Empire ${ }^{8}$

${ }^{5}$ Paris, 1958, rééd. 2000.

${ }^{6}$ Dans Past and Present, 46, 1970, p. 3-41; rééd. dans M.I. Finley (éd.), Studies in Ancient Society, Londres-Boston 1974, p. 166-209.

${ }^{7}$ Par exemple L. et J. RoBert, La Carie. Histoire et géographie historique, II, Paris, 1954, p. 319

${ }^{8}$ Oxford, 1969. 
et par C.P. Jones9. Une synthèse sur la question, à partir des inscriptions, a été récemment procurée par B. Puech ${ }^{10}$. Quand on examine de près la documentation historique, prosopographique, épigraphique, numismatique, ainsi que les sources littéraires elles-mêmes, on constate l'omniprésence des sophistes dans la société hellénophone. Il s'avère que les sophistes n'étaient pas seulement des hommes de savoir, mais aussi des hommes de pouvoir, ce pouvoir découlant, au moins en partie, de la maîtrise de la rhétorique qui était la leur ${ }^{11}$.

Cette deuxième approche a eu des répercussions sur la première, en ce qu'elle a conduit à réinterpréter de manière historique et anthropologique la culture des sophistes. Suivant cette réinterprétation, la mimêsis n'apparaît plus comme un passéisme scolaire, mais comme une mémoire culturelle, faisant sens dans le présent ${ }^{12}$, destinée à des publics bien définis ${ }^{13}$ et construisant une identité grecque. Le thème complexe de l'identité grecque dans l'Empire romain est un des sujets les plus fréquemment abordés, ces dernières années, à propos de la Seconde Sophistique, et il donne lieu à de multiples travaux ${ }^{14}$.

${ }^{9}$ The Roman World of Dio Chrysostom, Cambridge Mass.-Londres, 1978; Culture and Society in Lucian, Cambridge Mass.-Londres, 1986; Kinship Diplomacy in the Ancient World, Cambridge Mass.-Londres, 1999, chap. 9.

${ }^{10}$ Orateurs et sophistes grecs dans les inscriptions d'époque impériale, Paris, 2002.

${ }^{11}$ L. Pernot, "L'art du sophiste à l'époque romaine: entre savoir et pouvoir", dans C. Lévy, B. Besnier, A. Gigandet (éds.), Ars et ratio. Sciences, arts et métiers dans la philosophie hellénistique et romaine, Bruxelles, 2003, p. 126-142. Sur la fonction politique et sociale remplie par les discours épidictiques des sophistes, voir Id., La rhétorique de l'éloge dans le monde gréco-romain, Paris, 1993; Eloges grecs de Rome, Paris, 1997.

${ }^{12}$ G.A. Kennedy, The Art of Rhetoric in the Roman World, 300 B.C. - A.D. 300, Princeton, 1972; P. Desideri, Dione di Prusa. Un intellettuale greco nell'Impero romano, MessinaFirenze, 1978; G. Anderson, The Second Sophistic. A Cultural Phenomenon in the Roman Empire, Londres-New York, 1993; D. CAMPaniLE, "La costruzione del sofista. Note sul bios di Polemone", Studi ellenistici, 12, 1999, p. 269-315; Ead., "Vivere e morire da sofista: Adriano di Tiro", ibid., 15, 2003, p. 245-273.

${ }^{13}$ T. Schмiтz, Bildung und Macht. Zur sozialen und politischen Funktion der zweiten Sophistik in der griechischen Welt der Kaiserzeit, Munich, 1997; M. Korenjak, Publikum und Redner. Ihre Interaktion in der sophistischen Rhetorik der Kaiserzeit, Munich, 2000.

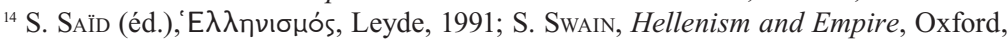
1996; S. Goldhill (éd.), Being Greek under Rome, Cambridge, 2001; T. Whitmarsh, Greek Literature and the Roman Empire, Oxford, 2001; E.N. Ostenfeld (éd.), Greek Romans and Roman Greeks, Aarhus, 2002. Important, à cet égard, l'article de P. VEYNE, "L'identité grecque devant Rome et l'Empereur", Revue des études grecques, 112, 1999, p. 510-567. Pour la problematique de l'identité sexuelle — qui n'est pas sans lien avec celle de l'identité grecque -, voir M.W. Gleason, Making Men. Sophists and Self-Presentation in Ancient Rome, Princeton, 1995; E. Gunderson, Staging Masculinity. The Rhetoric of Performance in the Roman World, Ann Arbor, 2000. 


\section{La Seconde Sophistique au seuil de l'Antiquité tardive}

Nous en arrivons à la piste qui forme l'essentiel de cette contribution. Après les sophistes de la mimêsis — hommes du passé —, après les sophistes dans l'Empire - hommes du présent — , voici les sophistes annonçant l'Antiquité tardive, les sophistes hommes de l'avenir. Les approches envisagées jusqu'ici donnent de la Sophistique une image scolaire, rationnelle, politique et publique. Cette image est juste, mais elle ne contient pas tout. Il faut envisager d'autres aspects, que nous présenterons en donnant des exemples. Le cas d'Aelius Aristide, qui fournit la documentation la plus riche, nous servira de fil directeur, et nous le complèterons grâce à des rapprochements avec les autres représentants de la Seconde Sophistique.

Il s'agit de mettre en lumière, dans la culture et les conceptions des sophistes des I $\mathrm{er}^{\mathrm{e}}-\mathrm{III} \mathrm{e}^{\mathrm{e}}$ siècles après J.-C., des traits qui sont plutôt associés, d'ordinaire, à l'Antiquité tardive. C'est le portrait d'une Seconde Sophistique inattendue qui va être tracé, en matière religieuse, philosophique et littéraire. En conclusion il faudra nous demander comment doivent être appréciées ces convergences entre Seconde Sophistique et Antiquité tardive et quelles conséquences il est permis d'en tirer.

\subsection{Aspects de l'expérience religieuse}

Parallèlement au respect affiché pour le Panthéon traditionnel et pour le culte impérial, la Seconde Sophistique connut des formes de dévotion intimes et exacerbées. Les œuvres d'Aelius Aristide offrent l'exemple saisissant d'une expérience de religion personnelle et de religion angoissée (pour reprendre deux mots illustrés, respectivement, par le P. Festugière et par E.R. Dodds) ${ }^{15}$, l'exemple d'un vécu exigeant et mystique, qui s'exprime avec force dans les Discours sacrés et dans les Hymnes. Aristide, on le sait, se voua tout entier au dieu Asclépios, duquel il reçut, sa vie durant, soutien et assistance. Il faut préciser que cette dévotion n'était pas déconnectée du métier de sophiste. Aristide n'était pas dévot d'un côté et sophiste de l'autre. Ces deux aspects étaient au contraire étroitement liés, car la protection d'Asclépios s'appliquait autant au talent oratoire et à la carrière publique d'Aristide qu'à sa santé physique. C'est en tant que sophiste qu'Aristide était le protégé d'Asclépios ${ }^{16}$.

${ }^{15}$ A.-J. Festugière, Personal Religion Among the Greeks, Berkeley-Los Angeles, 1954; E.R. Dodds, Pagan and Christian in an Age of Anxiety, Cambridge, 1965.

${ }^{16}$ L. Pernot, "Les Discours Sacrés d'Aelius Aristide entre médecine, religion et rhétorique", Atti dell'Accademia Pontaniana, n. s., 51, 2002, p. 369-383. 
Un autre sophiste des plus célèbres, Polémon de Laodicée, fréquenta comme Aristide le sanctuaire d'Asclépios à Pergame, et il y dédia une statue à Démosthène, à la suite d'une injonction reçue en rêve de la part du dieu ${ }^{17}$. Un troisième, Antiochos, se rendait au sanctuaire d'Asclépios situé dans sa patrie, à Aigeai, et y conversait avec le dieu, de nuit comme de jour ${ }^{18}$. On voit d'après ces exemples comment les sophistes pouvaient entretenir, ès qualités, des relations privilégiées avec les dieux.

De là au sacré, il n'y a qu'un pas. Le sophiste devint une variété de saint homme, de "holy man" doté de pouvoirs surnaturels : voici quelques illustrations de ce fait. Certaines œuvres d'Aristide portent le titre de "discours sacrés" (hieroi logoi) et de "discours oraculaires" (manteutoi), ce qui transforme leur auteur en une sorte de prophète ou d'interprète divin ${ }^{19}$. Polémon, grâce à sa maîtrise de la physiognomonie, était capable de prévoir l'avenir : ainsi, il put deviner que deux mariages qui se préparaient n'auraient pas lieu, simplement en observant le visage des fiancées (effectivement, l'une fut enlevée au beau milieu de la cérémonie, tandis que l'autre prit la fuite) ${ }^{20}$. Les sophistes Denys de Milet et Hadrien de Tyr passèrent pour des magiciens aux yeux de beaucoup, comme cela arriva par ailleurs à Apulée $e^{21}$. Le charme et la fascination exercés par les discours étaient considérés comme un pouvoir magique $^{22}$. Aristide parle de "drogue" (pharmakon) et de sortilège à ce propos ${ }^{23}$. Lucien compare la persuasion à un lien invisible qui tiendrait les auditeurs captifs à distance, enchaînés par les oreilles à la langue de l'orateur ${ }^{24}$. Favorinus, selon les sources, exerçait un pouvoir de séduction grâce à sa voix ${ }^{25}$. Tous ces exemples montrent que les sophistes étaient facilement considérés comme des "patrons" portant en eux une concentration de sacré.

Or le "holy man" est un type, un "style" qui sera particulièrement important dans l'Antiquité tardive, comme l'ont établi les travaux de L. Bieler

${ }^{17}$ C. Haвicht, Die Inschriften des Asklepieions (Altertümer von Pergamon, VIII, 3), Berlin, 1969, p. 75-76, n 33 = Puech, Orateurs et sophistes grecs cit., p. 399-401, n 210.

${ }^{18}$ Philostrate, Vies des sophistes, II, 4, 1 (568). Voir L. Robert, "De Cilicie à Messine et à Plymouth avec deux pierres errantes", Journal des savants, 1973, p. 184; Id., "Documents d'Asie Mineure", Bulletin de correspondance hellénique, 101, 1977, p. 124-125.

${ }^{19}$ Aelius Aristide, Or. 37 et 47-52 (voir en particulier Or. 48, 9).

${ }^{20}$ Voir G. Anderson, Philostratus, Londres, 1986, p. 142.

${ }^{21}$ Philostrate, Vies des sophistes, I, 22, 2 (523); II, 10, 6 (590); Apulée, Apologie.

${ }_{22}^{2}$ J. DE Romilly, Magic and Rhetoric in Ancient Greece, Cambridge Mass.-Londres, 1975, chap. IV.

${ }^{23}$ Aelius Aristide, Panathénaïque (1), 330; Défense de la rhétorique (2), 412; Contre les profanateurs (34), 26. 45.

${ }^{24}$ Lucien, Héraclès (5), 3. 5; Zeus tragédien (21), 45; Icaroménippe (24), 3; Le Scythe (68), 11.

${ }^{25}$ A. Barigazzi, Favorino di Arelate, Opere, Florence, 1966, p. 89 et suiv., test. 3-6. 
et de P. Brown ${ }^{26}$. Nous rencontrons donc là un trait qui rapproche la Seconde Sophistique de l'Antiquité tardive. P. Brown a signalé un tel rapprochement à propos des Discours sacrés d'Aelius Aristide, et rien n'est plus intéressant que les nuances avec lesquelles il s'exprime à ce sujet ${ }^{27}$.

Aristide avait le sentiment de vivre en contact direct et permanent avec son dieu Asclépios. Il recevait des visions. Il menait une vie recluse et faisait souffrir son corps, en refusant de s'alimenter, de se baigner, de se soigner normalement, et en se soumettant à des traitements douloureux. Il lui arriva même, par sa seule présence, d'arrêter un tremblement de terre ${ }^{28}$. Aussi estil tentant de le comparer aux ermites, aux membres de sectes ou aux saints qu'a connus l'Antiquité tardive. P. Brown a envisagé cette assimilation, mais il a fini par la refuser, parce que, écrit-il, la vie d'Aristide n'était pas tournée vers une transcendance; sa relation avec le divin était destinée à l'aider sur terre, non à lui assurer le salut dans l'au-delà.

L'embarras du savant irlandais tient au fait qu'il aperçoit distinctement des ressemblances importantes entre l'expérience religieuse vécue par Aristide et les styles de vie de l'Antiquité tardive, mais qu'il bute sur le problème de la transcendance. Il écrit : "Nous sommes obscurément irrités par le fait qu'un degré d'intimité avec le divin, qui aurait fait de l'un d'entre nous un saint ou un martyr, puisse servir simplement à produire un hypocondriaque d'une indomptable obstination. [...] Pour nous, le contact avec le divin doit être utilisé sur terre de façon beaucoup plus énergique". D'où cette image: "Aristide donne l'impression d'une puissante locomotive qui cale à force d'être conduite au frein".

Quant à nous, nous sommes surtout sensible aux éléments positifs et aux rapprochements dégagés par la brillante étude de P. Brown. Si la transcendance semble absente à un regard moderne (et encore faudrait-il vérifier ce point), c'est parce qu'Aristide n'était pas chrétien. Il manque la transcendance dans le ou les sens chrétiens de ce terme. Mais, pour le reste, les parentés avec l'Antiquité tardive existent sans aucun doute. Aristide était sur la voie (pour reprendre l'image du train), dans la direction de l'époque tardive, et il ne faut pas sous-estimer la force de ce mouvement. Quand P. Brown estime qu'Aristide n'avait pas rompu avec la société et qu'il n'osait pas se démarquer du style de vie de ses pairs, cette observation est justifiée

${ }^{26}$ L. Bieler, Theios anêr, Vienne, 1935-1936; P. Brown, Society and the Holy in Late Antiquity, Berkeley, 1982. Voir aussi G. Anderson, Sage, Saint and Sophist, Londres-New York, 1994, qui met l'accent, à juste titre, sur l'existence de "holy men" dès le début de l'époque impériale.

${ }^{27}$ Genèse de l'Antiquité tardive, Paris, 1978, p. 89-95 (trad. fr., par A. Rousselle, de The Making of Late Antiquity, Cambridge Mass., 1978).

${ }^{28}$ Aelius Aristide, Discours sacrés, III (49), 40. 
si l'on compare Aristide à certains de ses contemporains qui furent beaucoup plus marginaux que lui, mais elle ne tient peut-être pas assez compte d'une attitude de retrait, de différence et de supériorité affichée qui fut en réalité très accusée. Bien loin d'être incapable d'affirmer ouvertement sa proximité avec le divin, Aristide voulut faire connaître cette proximité, ainsi que le montrent différents passages de l'œuvre, et des Discours sacrés eux-mêmes, dans lesquels l'intimité avec Asclépios et les conséquences surnaturelles qui s'ensuivaient furent bel et bien révélées et proclamées.

Retenons une dernière formule de P. Brown: "Aelius Aristide marchait sur le fil du rasoir". Oui, parce qu'il était prêt à basculer dans l'Antiquité tardive; et peut-être avait-il déjà basculé plus que ne le reconnaît le critique.

Reste le problème du christianisme, auquel il vient d'être fait allusion. La Seconde Sophistique était majoritairement païenne. Toutefois, elle n'a pas ignoré le judaïsme et le christianisme, contrairement à ce que l'on croit parfois. Dion de Pruse loua les Esséniens, Lucien connut Pérégrinos (lequel fut chrétien pendant une partie de sa vie), le sophiste Amphiclès de Chalcis rédigea des imprécations influencées par le Deutéronome, le sophiste Rufinus rencontra à Smyrne le martyr Pionios ${ }^{29}$. Aristide et Ménandros évoquent la Palestine, l'auteur du traité $\mathrm{Du}$ sublime cite la Genèse ${ }^{30}$. Ces indices traduisent l'existence d'échanges - tantôt favorables, tantôt hostiles - entre la Seconde Sophistique et les milieux juifs et chrétiens. Les problématiques de rencontre culturelle et d'acclimatation entre paganisme et christianisme, qui seront si importantes dans l'Antiquité tardive, se laissent apercevoir dès les $\mathrm{I}^{\mathrm{er}}-\mathrm{III}{ }^{\mathrm{e}}$ siècles après J.-C. ${ }^{31}$

\subsection{Les idées philosophiques}

Dans le domaine des idées philosophiques également, la Seconde Sophistique se révèle proche de certaines tendances de l'Antiquité tardive. Indiquons, en préalable, que les sophistes n'étaient nullement coupés de la

${ }^{29}$ Synésios, Dion, 3, 1; Lucien, Sur la mort de Pérégrinos (55); L. Robert, "Malédictions funéraires grecques", Comptes rendus de l'Académie des Inscriptions et Belles-Lettres, 1978, p. 245-252; Id., Le Martyre de Pionios, prêtre de Smyrne, mis au point et complété par G.W. Bowersock et C.P. Jones, Washington, 1994, p. 98.

${ }^{30}$ Aelius Aristide, Pour les Quatre (3), 671; Ménandros le Rhéteur, Division des discours épidictiques, 366, 27; Ps.-Longin, Du sublime, 9, 9.

${ }^{31}$ Voir L. Pernot, "Saint Pionios, martyr et orateur", dans G. Freyburger - L. Pernot (éds.), Du héros païen au saint chrétien, Paris, 1997, p. 111-123; Id., "Christianisme et sophistique", dans L. Calboli Montefusco (éd.), Papers on Rhetoric, IV, Rome, 2002, p. 245-262. Dans le même sens: M. Rizzi, "Athéisme chrétien et primauté dans la cité", dans G. DorivaL - D. Pralon (éds.), Nier les dieux, nier Dieu, Aix-en-Provence, 2002, p. 279-292. 
philosophie, malgré une opinion courante. Au contraire, ils l'étudiaient et la pratiquaient $^{32}$. Ainsi, Alexandre de Séleucie, disciple de Favorinus, est cité comme sophiste par Philostrate et comme platonicien par Marc Aurèle ${ }^{33}$. Polémon suivit pendant quatre ans les leçons du philosophe Timocratès d'Héraclée et se rendit en Bithynie pour entendre Dion de Pruse ${ }^{34}$. Hérode Atticus avait eu pour maître le platonicien Taurus et et comptait parmi ses familiers les philosophes Lucius et Sextus ${ }^{35}$. Certains se "convertissaient" de la sophistique (ou de la rhétorique) à la philosophie, comme Dion de Pruse, Lucien, Marc Aurèle; certains, inversement, de la philosophie à la sophistique, comme Aristoclès de Pergame ${ }^{36}$. D'autres encore restaient à la fois sophistes et philosophes, comme Favorinus d'Arles ou même, en un sens, Maxime de Tyr. Il y avait interpénétration entre les deux domaines.

Or, la philosophie de cette époque comprenait des thèmes porteurs d'avenir. Dans ce que C. Moreschini a appelé, à juste titre, "la culture philosophique du pepaideumenos", figurent la tendance à l'hénothéisme, appuyée sur une démonologie, le platonisme, la réflexion sur la prière, ou encore, dans la Vie d'Apollonios de Tyane de Philostrate (le biographe des sophistes), l'héliolâtrie et la théosophie ${ }^{37}$. Ces conceptions philosophiques, qui avaient d'ailleurs des implications religieuses, annoncent des thèmes que l'on rencontre dans le néoplatonisme et dans le christianisme.

Ici encore, on peut citer l'exemple d'Aelius Aristide, en faisant référence aux Discours platoniciens de cet auteur. Cet imposant ensemble, qui occupe quatre cents pages de texte grec, a pour but de répondre aux critiques que Platon avait portées contre la rhétorique, dans le Gorgias en particulier. En apparence, il s'agit d'une discussion d'intérêt purement historique, voire passéiste, ressortissant à la mimêsis. C'est ainsi que le texte a généralement été lu, jusqu'à une étude récente qui a renouvelé la perspective ${ }^{38}$. A.M. Milazzo a montré que les Discours platoniciens posent des problèmes qui étaient vivants et importants dans la culture de l'époque, tant chez les païens que chez les chrétiens, et qui étaient appelés à connaître une importance encore plus

${ }^{32}$ Voir B. Cassin, L'effet sophistique, Paris, 1995; G.W. Bowersock, "Philosophy in the Second Sophistic", dans G. Clark, T. RaJak (éds.), Philosophy and Power in the GraecoRoman World, Oxford, 2002, p. 157-170.

${ }^{33}$ Philostrate, Vies des sophistes, II, 5; M. Aurèle, Ecrits pour lui-même, I, 12.

${ }^{34}$ Philostrate, ibid., I, 25, 5 (535-536). 8 (539). 9 (541).

${ }^{35}$ Philostrate, ibid., II, 1, 14 (564). 8-10 (556-558).

${ }^{36}$ Philostrate, ibid., II, 3 (567).

${ }^{37}$ C. Moreschini, "Aspetti della cultura filosofica negli ambienti della Seconda Sofistica", Aufstieg und Niedergang der römischen Welt, II, 36,7, 1994, p. 5101-5133.

${ }^{38}$ A.M. Milazzo, Un dialogo difficile : la retorica in conflitto nei Discorsi Platonici di Elio Arisitde, Hildesheim, 2002. 
grande dans les siècles suivants : problèmes de l'interprétation de Platon, des rapports entre philosophie et rhétorique, entre paganisme et christianisme, de la philosophie comme mode de vie, du dialogue et de la médiation entre les disciplines. Les Discours platoniciens d'Aristide doivent être appréciés au moins autant par rapport à l'Antiquité tardive, qu'ils préfigurent, que par rapport à l'Athènes classique, sur laquelle ils portent apparemment.

Cette situation particulière se traduit notamment dans un passage où le sophiste compare Démosthène à Hermès Logios ${ }^{39}$. En citant Démosthène, Aristide se réfère au modèle classique de la rhétorique, à l'Orateur par excellence tel que le définissent la paideia et la mimêsis. Mais en faisant intervenir Hermès Logios comme point de comparaison, Aristide ouvre sa démonstration à une conception mystique de la rhétorique et à l'hermétisme. C'est pourquoi cette comparaison a intéressé les auteurs de l'Antiquité tardive (notamment les néoplatoniciens), qui l'ont abondamment citée et recyclée.

\subsection{Les formes littéraires}

Enfin, il convient de répertorier, de façon nécessairement cursive, un certain nombre de formes littéraires qui sont associées à l'Antiquité tardive, et dont on peut montrer qu'elles furent mises en œuvre dès l'époque de la Seconde Sophistique.

Aelius Aristide fournit l'exemple des récits de rêves (toujours dans les Discours sacrés), auxquels s'adjoint le traité sur l'interprétation des rêves de son contemporain Artémidore de Daldis. La conception du rêve comme "oracle privé" ${ }^{40}$ et la rédaction d'ouvrages de toutes sortes à ce sujet est un trait de la Seconde Sophistique, bien avant l'époque de Synésios. Les rêves, on le sait, jouent aussi un rôle important dans les romans.

Le roman, précisément, est une de ces formes littéraires qui ont longtemps été considérées comme tardives (en tout cas du côté grec) et que les progrès de la recherche ont notablement vieillies. Les découvertes papyrologiques, notamment, ayant fait remonter la datation des principaux romans grecs, l'on s'aperçoit à présent que le genre romanesque a été largement pratiqué à l'époque de la Seconde Sophistique, et par des auteurs qui étaient euxmêmes sophistes ou proches de la sophistique et de la rhétorique : Lucien, auteur de L'âne; Lollianos, auteur des Phoinikika et peut-être identique au

${ }^{39}$ L. Pernot, “' 'L'empreinte d'Hermès Logios': une citation d'Aelius Aristide chez Julien et chez Damascius", Rendiconti dell'Accademia di archeologia, lettere e belle arti, 71, 2002, p. 191-207; Id., L'ombre du Tigre. Recherches sur la réception de Démosthène, Naples, sous presse, chap. III.

${ }^{40}$ P. Brown, Genèse de l'Antiquité tardive cit., p. 87. 
sophiste Lollianos de Philostrate; Chariton secrétaire d'un rhêtôr. Il y aurait aujourd'hui une étude à écrire sur les rapports entre les romans grecs et la rhétorique de la Seconde Sophistique.

L'autobiographie, illustrée à l'époque tardive par Libanios, Grégoire de Nazianze, saint Augustin, etc., a existé dès l'époque de la Seconde Sophisti$q^{4} e^{41}$. Dans les Discours sacrés d'Aelius Aristide ou dans le Songe de Lucien, l'autobiographie se lie d'ailleurs au thème du rêve. Chez Dion de Pruse, on a affaire à une mise en scène du "moi". Plus bruts, probablement, devaient être les mémoires (ephêmerides) composés par Hérode Atticus.

La poésie, qui pourrait paraître absente des préoccupations des sophistes, en réalité ne leur était pas étrangère. Scopélien composa une épopée, Aristide des hymnes, Hippodromos des nomes lyriques, le premier Philostrate des tragédies et des comédies, Isagoras (élève de Chrestos de Byzance) des tragédies, Lucien des pastiches de tragédie ${ }^{42}$, sans compter tous ceux qui composèrent des épigrammes. La domination de la rhétorique éclipsait cette production poétique, et les aléas de la transmission font que les textes ne nous sont pas parvenus. Mais il n'en est pas moins nécessaire de reconnaître, dans le paysage de la sophistique, la présence de la poésie, composée, chantée, représentée.

L'épistolographie fut pratiquée par les sophistes, le meilleur exemple étant offert par Hérode Atticus, dont la correspondance était encore considérée, un siècle après sa mort, comme le modèle du genre ${ }^{43}$. Un autre exemple, sur le versant latin, serait la correspondance de Fronton. A ce propos il faut renvoyer aux pages d'A. Garzya sur "L'epistolografia letteraria tardoantica", essai paru dans $\mathrm{Il}$ mandarino e il quotidiano ${ }^{44}$. Au début de l'article, l'auteur écrit: "Cominciamo intanto col chiarire in quali termini sia storiograficamente legittimo configurare un, per cosí dire, periodo tardoantico - dal II,

${ }^{41}$ Philostrate, Vies des sophistes, II, 1, 14 (565). Voir en général M.-F. BASLEZ - P. Hoffmann - L. Pernot (éds.), L'invention de l'autobiographie, d'Hésiode à saint Augustin, Paris, 1993.

${ }^{42}$ Respectivement, Philostrate, Vies des sophistes, I, 21, 5 (518); Aelius Aristide, fragments 23-44 Behr; Philostrate, ibid., II, 27, 6 (620); Souda, F 422; Philostrate, ibid., II, 11, 1 (591); Lucien, La tragédie de la goutte (69). Voir les recherches menées par E.L. BowIE sur la poésie de cette époque: "Poetry and Poets in Asia and Achaia", in S. WALKER - Av. CAmeron (éds.), The Greek Renaissance in the Roman Empire, Londres, 1989, p. 198-205; "Greek Sophists and Greek Poetry in the Second Sophistic", Aufstieg und Niedergang der römischen Welt, II, 33, 1, 1989, p. 209-258; "Greek Poetry in the Antonine Age", in D.A. Russell (éd.), Antonine Literature, Oxford, 1990, p. 53-90; "Hadrian and Greek Poetry", in E.N. Ostenfeld (éd.), Greek Romans and Roman Greeks cit., p. 172-197.

${ }^{43}$ Philostrate, Vies des sophistes, I, 8, 3 (490); I, 25, 7 (537); II, 1, 7 (552). 13 (563). 14 (565); Dialexis I, vol. II, p. 258, 5-8 Kayser.

${ }^{44}$ Naples, 1983, p. 113-148 (p. 117 pour la citation). 
intendiamo, al VII secolo - nella storia della epistolografia greco-latina". Cette précision chronologique, "dal II, intendiamo, al VII secolo", est significative pour la présente démonstration. Elle a pour effet d'annexer le deuxième siècle à l'Antiquité tardive, ou en tout cas de montrer qu'il n'y a pas de coupure, dans le domaine de l'épistolographie, entre le deuxième siècle et l'Antiquité tardive, ce qui concorde avec l'hypothèse présentée ici.

Les observations qui ont été avancées enrichissent, nous l'espérons, la description que l'on donne ordinairement de la Seconde Sophistique. Il apparaît que ce mouvement ne se réduisait pas à la mimêsis et à l'importance politique des sophistes. Il comportait aussi des expériences de mysticisme, une culture philosophico-religieuse, des rapports avec le judaïsme et le christianisme, une pratique littéraire riche et variée s'étendant, au-delà des discours rhétoriques, à la poésie, à l'autobiographie, à l'épistolographie. On pourrait ajouter encore, si l'espace imparti le permettait, la diffusion du bilinguisme grec-latin, plus répandu qu'on ne l'admet d'ordinaire, et la fréquence des contacts inter-ethniques et inter-culturels sur toute l'étendue de l'Empire. C'est ce foisonnement de traits qui autorise à rapprocher la Seconde Sophistique de l'Antiquité tardive.

Pour assurer la validité de ce rapprochement, il faut évoquer brièvement quelques questions soulevées par la présente analyse:

Une question concerne la place occupée par Aelius Aristide dans la démonstration. Cet auteur fournit beaucoup d'exemples. Est-ce à dire que la démonstration ne vaut que pour lui ? Non, car, remarquons-le, il n'est jamais isolé : sur chaque point, d'autres sophistes sont toujours, également, allégués. Si Aristide est souvent cité, c'est parce que son œuvre est conservée, à la différence de celle des autres sophistes. Quelles que fussent les bizarreries de son idiosyncrasie, Aristide était bel et bien représentatif de la Seconde Sophistique. Grâce au fait que ses discours ont été transmis, il se révèle une figure-clé dans le passage de la Seconde Sophistique vers l'Antiquité tardive.

Une deuxième question tient au rapport entre les éléments traditionnels de la description de la Seconde Sophistique (mimêsis et influence politique) et les éléments de description que nous avons tenté d'ajouter (en matière religieuse, philosophique et littéraire). Les éléments traditionnels pourraient donner lieu, eux aussi, à des rapprochements avec l'Antiquité tardive. Car l'Antiquité tardive a connu, évidemment, la mimêsis, elle a connu le rôle politique des intellectuels. Si nous avons choisi d'insister sur les autres aspects, c'est parce qu'ils paraissent plus caractéristiques, plus spécifiques de l'époque tardive, et c'est pourquoi ils méritent de retenir l'attention dans le contexte de la présente discussion. 
Il faut enfin souligner que, naturellement, chaque élément n'est pas décisif par lui-même. Ce n'est pas parce que des sophistes ont écrit des poèmes qu'il y a parenté entre la Seconde Sophistique et l'Antiquité tardive. C'est l'ensemble du tableau qui est significatif. Il s'agit de comparer deux continents - Seconde Sophistique, Antiquité tardive —, à un niveau délibérément général (même si la démonstration repose sur des preuves précises), afin de réfléchir à l'esprit de chaque époque, au Zeitgeist.

$\mathrm{Si}$, compte tenu de ces remarques, on accepte notre hypothèse, il en découle des conséquences importantes. Nous sommes invités à considérer la Seconde Sophistique d'une manière quelque peu différente : il ne faut pas s'en tenir à l'image officielle qui est donnée de ce mouvement, une image centrée sur l'enseignement de la rhétorique et sur l'exercice de fonctions publiques (on dirait en France une image "Troisième République", c'est-à-dire dans laquelle l'accent est mis sur l'Ecole et l'Etat), et il faut admettre, chez les sophistes, l'existence d'aventures individuelles, d'expériences irrationnelles, de tout une complexité intellectuelle et littéraire. S'il est juste de dire que cette complexité ouvre sur l'avenir, dès lors, c'est la délimitation même du mouvement qui est en cause. Faut-il borner la Seconde Sophistique au III' siècle, comme on le fait le plus souvent? Ne faut-il pas plutôt reconnaître que la Seconde Sophistique se continue au IV siècle et au-delà (avec des différences et des évolutions, naturellement) ${ }^{45}$ ? Parlera-t-on d'une Seconde Sophisitique bis ou d'une Troisième Sophistique à partir du IV siècle ?

Par ailleurs, le pont jeté entre la Seconde Sophistique et l'Antiquité tardive est une contribution à la réflexion sur la genèse de l'Antiquité tardive. Le problème qui se pose, en particulier, est celui de la périodisation ${ }^{46}$. On peut se demander si, du point de vue culturel, les racines de l'Antiquité tardive ne sont pas à chercher dans le ${ }_{\text {II }} \mathrm{e}$ siècle.

Telles sont les questions qui se posent, et qui doivent encore être approfondies. Pour conclure, nous citerons un dernier exemple. Il s'agit de Titus Flavius Glaucos de Marathon, personnage du milieu du III siècle après J.C., connu par des inscriptions d'Athènes, d'Eleusis et d'Olympie et par des épigrammes conservées dans l'Anthologie Palatine ${ }^{47}$. Issu d'une importante

${ }^{45}$ L. Cracco RugGini évoque "gli sviluppi della sofistica nell'età postseveriana", dans "Sofisti greci nell'Impero romano", Athenaeum, 49, 1971, p. 415. Dans le même sens, voir G.A. Kennedy, Greek Rhetoric under Christian Emperors, Princeton, 1983, qui inclut un chapitre sur "The Schools of the Sophists" dans son exposé consacré à l'époque tardive (tout en marquant des différences par rapport à l'époque antonine).

${ }^{46}$ Voir sur ce problème les intéressantes réflexions de H. Inglebert, "Peter Brown", dans V. Sales (éd.), Les historiens, Paris, 2003, p. 344-345.

${ }^{47}$ Voir B. Puech, "Glaucos (T. Flavius) de Marathon", Dictionnaire des philosophes antiques, III, Paris, 2000, p. 485; Ead., Orateurs et sophistes grecs cit., p. 270-283, 516-526. 
famille de l'Attique, qui comptait des hiérophantes d'Eleusis et des prêtres d'Asclépios et qui avait des alliances dans l'ordre sénatorial, Glaucos était l'arrière-petit-fils du poète et philosophe stoïcien Sarapion (ami de Plutarque) et le neveu du philosophe et élève des sophistes Callaischros. Sur une inscription de l'Asclépieion d'Athènes, élevée en l'honneur de Quintus Statius Thémistoclès, Glaucos se définit lui-même comme "poète, orateur et philosophe" (poiêtês kai rhêtôr kai philosophos), et également "ancien avocat du fisc" (apo sunêgoriôn tamiou). Quant au Thémistoclès de l'inscription, il est possible qu'il soit identique au stoïcien homonyme évoqué par Porphyre dans la Vie de Plotin et célébré par un oracle de Delphes ${ }^{48}$.

Glaucos se trouvait ainsi au carrefour des époques et des cultures. Par son bisaïeul Sarapion, il remontait jusqu'à l'époque de Plutarque, tandis que par le jeune Thémistoclès il descendait jusqu'à l'époque de Porphyre. Par sa qualité d'orateur et d'avocat du fisc, il appartenait au monde de la rhétorique et de l'influence politique, mais il était aussi poète, philosophe et fervent d'Asclépios. Titus Flavius Glaucos de Marathon est une figure emblématique, qui illustre la présence, au sein de la Seconde Sophistique, d'éléments qui annoncent l'Antiquité tardive, selon le processus de continuité que nous avons essayé de décrire.

NotA Bene. Une version italienne de ce texte paraît dans les actes du VIe convegno nazionale de l'Associazione di Studi Tardoantichi, "Forme della cultura nella Tarda Antichità", édités par Ugo Criscuolo à Naples.

${ }^{48}$ Puech, Orateurs et sophistes grecs cit., p. 526; R. Goulet, Etudes sur les Vies de philosophes de l'Antiquité tardive, Paris, 2001, p. 207. 
RÉSumÉ. Les chercheurs n'ayant guère songé à jeter des ponts entre la Seconde Sophistique et l'Antiquité tardive, il s'agit d'ouvrir une piste, en posant la question de savoir dans quelle mesure le concept d'Antiquité tardive peut servir à interpréter de manière nouvelle la Seconde Sophistique. Les deux principales directions de la recherche sur la Seconde Sophistique ont consisté, d'une part, à mettre en relief la culture des sophistes et leurs références au passé grec de l'époque archaïque et classique, d'autre part, à souligner l'importance du rôle politique et social joué par les sophistes en leur temps. La troisième direction, qui est proposée ici, permet de faire apparaître, dans la culture et les conceptions des sophistes des ier-iiie siècles après J.-C., des traits qui annoncent l'Antiquité tardive et qui étaient, à l'époque, porteurs d'avenir. La Seconde Sophistique ne se limitait pas à l'admiration pour le passé et aux fonctions officelles exercées par les sophistes. Elle comportait aussi des formes de dévotion intimes et exacerbées, des expériences de mysticisme, un modèle de "holy man" doté de pouvoirs surnaturels, toute une culture philosophico-religieuse, des rapports avec le platonisme, avec le judaïsme et le christianisme, et encore une pratique littéraire riche et variée s'étendant, au-delà des discours rhétoriques, à la poésie, à l'autobiographie, à l'épistolographie, au récit de rêves, au roman.

Mots-Clés. Aelius Aristide; Antiquité tardive; littérature; philosophie; religion; rhétorique; Seconde Sophistique; Titus Flavius Glaucos de Marathon. 